\title{
Some Experiences of the use of Iterative Learning Control for Performance Improvement in Robot Control Systems
}

\author{
Svante Gunnarsson and Mikael Norrlöf \\ Department of Electrical Engineering \\ Linköping University, S-581 83 Linköping, Sweden \\ www: http://www.control.isy.liu.se \\ email: svante@isy.liu.se, mino@isy.liu.se
}

1997-06-04

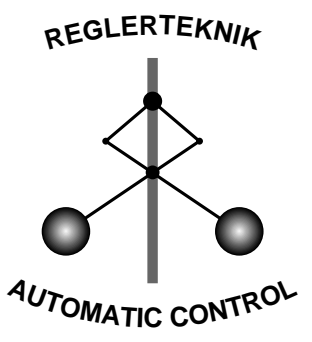

\section{LINKÖPING}

Technical reports from the Automatic Control group in Linköping are available by anonymous ftp at the address 130.236.20.24 (ftp.control.isy.liu.se/pub/Reports/). This report is contained in the compressed postscript file 1956.ps.Z. 


\title{
SOME EXPERIENCES OF THE USE OF ITERATIVE LEARNING CONTROL FOR PERFORMANCE IMPROVEMENT IN ROBOT CONTROL SYSTEMS
}

\author{
S.Gunnarsson and M. Norrlöf \\ Division of Automatic Control, Department of Electrical Engineering, Linköping \\ University, S-58183 Linköping, Sweden
}

\begin{abstract}
Some aspects of the use of learning control for improved performance in robot control systems are studied. The learning control signal is used in combination with conventional feed-back and feed-forward control. The effects of disturbances, unmodeled dynamics and friction are studied theoretically and in simulations of a simplified model of a robot arm. Convergence and robustness aspects of the choice of filters in the updating scheme of the learning control signal are studied.
\end{abstract}

Keywords. Robotics, Iterative Learning Control, Friction, Disturbance signals

\section{INTRODUCTION}

Learning control has been an active research area for more than a decade, mainly inspired by the pioneering work of Arimoto et al, (Arimoto et al. 1984). Due to the repetitive nature of many operations in robotics this has been a main area of interest in research on and applications of learning control, as discussed in, for example, (Craig 1988), (Horowitz 1993) and (Panzieri and Ulivi 1995). An important issue in learning control is the convergence properties of the learning procedure. This issue was addressed already in (Arimoto et al. 1984) and further studied in, for example, (Heinzinger et al. 1992), (Hideg 1992), (Liang and Looze 1993) and (Amann et al. 1995).

In this paper we shall consider iterative learning control as a complement to conventional feed-forward and feedback control. We shall mainly consider linear systems, but also study the effects of non-linear friction. The aim is to illustrate the fundamental properties of the ILC algorithm applied in this framework, with focus on convergence, robustness and disturbance effects.

\section{THE CONTROL PROBLEM}

Consider the problem of controlling a SISO linear system

$$
Y=G(U+D)
$$

where $U, Y$ and $D$ represent input, output and load disturbance respectively, and $G$ is the transfer function. Capital letters indicate that we work with transformed signals, and the discussion covers both continuous time and discrete time signals unless otherwise stated. The system is controlled by combined feed-forward and feedback using

$$
U=F_{f} Y_{D}+F\left(Y_{D}-(Y+N)\right)
$$

where $Y_{D}$ and $N$ denote the reference signal and a measurement disturbance respectively. Furthermore $F_{f}$ and $F$ denote the transfer functions of the feed-forward and the feed-back regulators. We shall consider the control signal generated by the feed-back regulator as error signal, i.e.

$$
E=F\left(Y_{D}-Y\right)
$$

In robot applications $F$ is often a PD-regulator, which means that the error signal will be a combination of the position error and the velocity error. We then get

$$
E=G_{C}\left(\left(G^{-1}-F_{f}\right) Y_{D}-D+F N\right)
$$

where $G_{C}$ is the transfer function of the closed loop system, i.e.

$$
G_{C}=\frac{F G}{1+F G}
$$

\section{ITERATIVE LEARNING CONTROL}

The use of feed-forward and feed-back control will in many cases give very good properties of robot control systems, but in order to further improve the performance we shall consider the use of learning control. We therefore add a correction signal $\Delta U_{k}$ to the input torque according to Figure 1. The input signal will thus be given by

$$
U_{k}=F_{f} Y_{D}+F\left(Y_{D}-\left(Y_{k}+N_{k}\right)\right)+\Delta U_{k}
$$




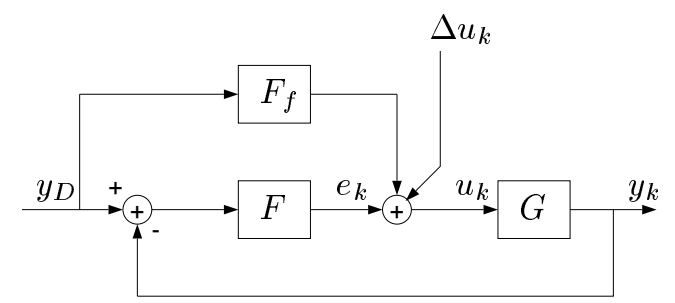

Fig. 1. A control system with feed-forward, feed-back and an ILC correction signal

where the index $k$ denotes the iteration number.

Considering only linear operations the updating of the correction signal can, in the frequency domain, be expressed as

$$
\Delta U_{k+1}=\sum_{j=0}^{k} \bar{H}_{j} E_{j}
$$

where $\bar{H}_{j}, j=0, \ldots, k$ are linear filters. For convenience we shall here however consider recursive update equations on the form

$$
\Delta U_{k+1}=H_{1} \Delta U_{k}+H_{2} E_{k}
$$

where $H_{1}$ and $H_{2}$ are linear filters. The choice of the filters $H_{1}$ and $H_{2}$ is a main task when designing a learning control algorithm, since the filters determine the convergence and robustness properties. One method for choosing appropriate filters in the update equation is presented in (de Roover 1996) where methods from design of robust controllers are applied. The filters are designed to give a convergent ILC algorithm despite uncertainties in the process model. In (Gorinevsky et al. 1995) the problem is considered form a different viewpoint and the choice of the ILC input signal is formulated as an optimization problem, resulting in a time domain updating equation for the input signal.

\section{CONVERGENCE PROPERTIES}

We shall now investigate how the error signal behaves when the update equation (8) is applied. Let us first introduce the signal $E_{0}$ defined by

$$
E_{0}=G_{C}\left(\left(G^{-1}-F_{f}\right) Y_{D}\right.
$$

which is the (disturbance free) error signal obtained in the first iteration when no correction signal is added, i.e. $\Delta U_{0} \equiv 0$. This gives

$$
\begin{aligned}
E_{k+1} & =E_{0}-G_{C} H_{1} \Delta U_{k}-G_{C} H_{2} E_{k} \\
& -G_{C} D_{k+1}+F G_{C} N_{k+1}
\end{aligned}
$$

and by adding and subtracting relevant terms on the right hand side we get

$$
\begin{aligned}
E_{k+1} & =\left(1-H_{1}\right) E_{0}+H_{1}\left(E_{0}-G_{C} \Delta U_{k}-G_{C} D_{k}\right. \\
& \left.+F G_{C} N_{k}\right)-G_{C} H_{2} E_{k}+H_{1} G_{C} D_{k} \\
& -G_{C} D_{k+1}-H_{1} F G_{C} N_{k}+F G_{C} N_{k+1}
\end{aligned}
$$

which implies the following error update equation

$$
\begin{aligned}
E_{k+1} & =\left(1-H_{1}\right) E_{0}+\left(H_{1}-G_{C} H_{2}\right) E_{k} \\
& +G_{C}\left(H_{1} D_{k}-D_{k+1}\right)+F G_{C}\left(N_{k+1}-H_{1} N_{k}\right)
\end{aligned}
$$

A corresponding equation is presented in (Panzieri and Ulivi 1995) for the open loop case and for load disturbances only.

The convergence properties are determined by the homogeneous part of the difference equation (13) and referring to (Craig 1988) the convergence condition, in the continuous-time case, is that

$$
\left|H_{1}(i \omega)-H_{2}(i \omega) G_{C}(i \omega)\right|<1 \quad \forall \omega
$$

Provided that the learning procedure converges the error signal becomes

$$
\bar{E}=\frac{1-H_{1}}{1-H_{1}+G_{C} H_{2}} E_{0}
$$

We see that by using $H_{1} \neq 1$ we are not able to eliminate the error completely, but as will be seen later other advantages are obtained by this choice. In e.g. (Owens 1993) the case $H_{1}=\mu$, where $\mu<1$ is a scalar, is studied. An alternative parameterization of the filters in the learning law was presented in (Mita and Kato 1985), where

$$
H_{1}=\frac{1}{1+V} \quad H_{2}=\frac{1}{1+V} H
$$

and where $V$ and $H$ are filters. In (Mita and Kato 1985) this configuration is used with $H$ scalar. The condition for convergence now becomes

$$
\left|1-H(i \omega) G_{C}(i \omega)\right|<|1+V(i \omega)|
$$

and it is obvious that the stability region can be extended by a suitable choice of the filter $V$, resulting in a so called stabilizing circle. By letting $V$ be frequency dependant the stability region can be extended in a frequency dependant way.

\section{DISTURBANCE EFFECTS}

A number of observations can be made using equation (13). Let us first consider the case $H_{1}=1$, which implies the update equation

$$
\begin{aligned}
E_{k+1} & =\left(1-G_{C} H_{2}\right) E_{k}+G_{C}\left(D_{k}-D_{k+1}\right) \\
& +F G_{C}\left(N_{k+1}-N_{k}\right)
\end{aligned}
$$


The disturbances contribute to the error equation by their differences between the iterations. If a disturbance is of repetitive nature in the sense that the disturbance signals $d_{k}(t)=d_{k+1}(t)$ and $n_{k}(t)=n_{k+1}(t)$ for all $k$ the contribution to the error difference equation is zero. This assumption is more likely for the load disturbance where for example load disturbances due to gravitational forces can be expected to be rather similar during different iterations. Measurement disturbances, on the other hand, are more likely to be of random character which means that $n_{k+1}(t) \neq n_{k}(t)$ in general, and there will hence always be a driving term on the right hand side of equation (18) that prevents $E_{k}(s)$ from tending to zero.

Let us also consider the situation with $H_{1} \neq 1$, neglect measurement disturbances and assume that $d_{k}(t)=d(t)$ $\forall k$. This corresponds to the error difference equation

$$
\begin{aligned}
E_{k+1} & =\left(1-H_{1}\right) E_{0}+\left(H_{1}-G_{C} H_{2}\right) E_{k} \\
& -G_{C} D\left(1-H_{1}\right)
\end{aligned}
$$

The load disturbance will act as a driving term similar to the initial error $E_{0}$.

\section{SIMULATION EXAMPLE}

\subsection{System description}

We shall consider a simplified description of a single robot joint modeled as a double integrator, i.e.

$$
G(s)=\frac{1}{J s^{2}}
$$

Since the system is computer controlled we shall use the discrete time representation given by the transfer function

$$
G(z)=\frac{T^{2}(z+1)}{2 J(z-1)^{2}}
$$

where $J=0.0094$ is the moment of inertia. The system is controlled by a discrete time PD-regulator given by

$$
F(z)=K_{P}+\frac{K_{D}}{T} \frac{(z-1)}{z}
$$

where $K_{P}=12.7$ and $K_{D}=0.4$. The feed-forward filter is chosen as a double differentiation represented by

$$
F_{f}=\frac{2 J^{*}(z-1)^{2}}{T^{2} z^{2}}
$$

where $J^{*}$ is the estimated moment of inertia. The correction signal will be updated according to equation (8) where $H_{1}(z)$ and $H_{2}(z)$ are filters that both may be non-causal. The model is simulated using $1 \mathrm{kHz}$ sampling frequency. For evaluation of the algorithm we shall apply the reference trajectory shown in Figure 2. This is a comparatively smooth trajectory and in real robot applications trajectories with more sharp acceleration profiles can be expected.

\subsection{Unmodeled dynamics}

The first goal is to investigate how the learning control approach can deal with unmodeled dynamics. We shall consider the case when there is an $30 \%$ error in $J^{*}$, i.e. the control system is based on an incorrect value of the moment of inertia. For $H_{1}(z)=1$ the ideal choice of $\mathrm{H}_{2}$ would be to choose it as the inverse of $G_{C}(z)$, which, theoretically, would result in convergence to zero in one step. This is however an unrealistic choice since it requires exact knowledge of the system and results in a filter with very high gain for high frequencies. Instead we consider

$$
H_{2}(z)=\hat{G}_{C}^{-1}(z)\left(1-H_{B}(z)\right)
$$

where $\hat{G}_{C}(z)$ denotes the closed loop transfer function we obtain by using the model of the open loop system and $H_{B}(z)$ is a Butterworth high pass filter (here of second order) for which the gain tends to one for high frequencies. Choosing $H_{2}(z)$ according to this design rule, with cut-off frequency of the high pass filter equal to 0.4 times the Nyquist frequency, gives the Nyquist curve depicted in Figure 3. Figure 3 also shows $G_{C}(z)$ for comparison. The whole Nyquist curve is now inside the learning circle while it for large frequencies tends to the origin. The learning control algorithm is then tested in simulations. Figure 4 shows the FFT of the error signal $e_{k}(t)$ for different iterations.

\subsection{Friction}

Since all robots contains some amount of friction it is of interest to evaluate the performance of the learning control algorithm under such conditions. The dynamics of the robot is then described by

$$
J \ddot{y}(t)=u(t)-f \operatorname{sign}(\dot{y}(t)) \quad \dot{y}(t) \neq 0
$$

and

$$
J \ddot{y}(t)=0 \quad|u(t)| \leq f, \quad \dot{y}(t)=0
$$

where the coefficient $f$ is chosen such that the friction force corresponds to $30 \%$ of the maximum torque, in this case 0.12 . The linear analysis carried out above is not applicable when we have introduced nonlinear elements into the problem but we can still evaluate the learning control algorithm using simulations. If we carry out the same simulations as in the previous case we get the result shown in Figure 5. 
Even though the filter $H_{2}(z)$ designed above was robust enough to handle that it was designed based on an incorrect value of the moment of inertia it is of interest to further improve the stability margins of the learning control algorithm. This can be done by using the filter $V(z)$ discussed above. In the simulations we have chosen $V\left(e^{i \omega}\right)$ as a first order high pass filter with cut-off frequency 0.7 times the Nyquist frequency. The high frequency gain of the filter is 0.1 , which means that the stability region is extended in the high frequency regions. The result of this choice is shown in Figure 3, where the obtained stabilizing circle is shown. In Figure 6 the simulations results are shown. The convergence properties are comparable with what was obtained without the use of $V\left(e^{i \omega}\right)$ and the error converges to approximately the same level. We have hence achieved the improved robustness without any significant increase in the error level.

\section{CONCLUSIONS}

The potential of learning control as a way of improving the performance of robot control systems has been investigated. Convergence and robustness aspects of the choice of design filters have been discussed. The proposed update method of the learning control signal works well also in the presence of nonlinear friction.

\section{ACKNOWLEDGMENTS}

This work was supported by CENIIT at Linköping University and ABB Robotics within ISIS at Linköping University.

\section{REFERENCES}

Amann, N., D.H. Owens and E. Rogers (1995). "Iterative Learning Control for Discrete Time Systems with Exponential Rate of Convergence". Technical report. Report Number: 95/14, Centre for Systems and Control Engineering, University of Exeter. Exeter, United Kingdom.

Arimoto, S., S. Kawamura and F. Miyazaki (1984). "Bettering Operation of Robots by Learning". Journal of Robotic Systems pp. 123-140.

Craig, J. (1988). Adaptive Control of Mechanical Manipulators. Addison-Wesley Publishing Company.

de Roover, D. (1996). "Synthesis of a Robust Iterative Learning Controller Using an $H_{\infty}$ approach". In: Proc. 35th CDC. Kobe, Japan. pp. 3044-3049.
Gorinevsky, D.M., D. Torfs and A.A. Goldenberg (1995). "Learning approximation of feedforward dependence on the task parameters: Experiments in direct-drive manipulator tracking". In: Proc. $A C C$ 1995. Seattle, Washington. pp. 883-887.

Heinzinger, G., D. Fenwick, B. Paden and F. Miyazaki (1992). "Stability of Learning Control with Disturbances and Uncertain Initial Conditions". IEEE Trans. Automatic Control 37, 110-114.

Hideg, L. (1992). Stability of Learning Control Systems. PhD thesis. Oakland University. Rochester, Michigan.

Horowitz, R. (1993). "Learning Control of Robot Manipulators". ASME Journal of Dynamic Systems, Measurement, and Control 115, 403-411.

Liang, Y.J. and D.P Looze (1993). "Performance and Robustness Issues in Iterative Learning Control". In: Proc. 32nd CDC. San Antonio, TX. pp. 19901995 .

Mita, T. and E. Kato (1985). "Iterative Control and its Application to Motion Control of Robot Arm - A Direct Approach to Servo-Problems". In: Proc. 24th $C D C$. Ft. Lauderdale, Florida. pp. 1393-1398.

Owens, D. H. (1993). "2D Systems Theory and Iterative Learning Control". In: Proc. 2nd European Control Conference. Groningen, Holland. pp. 1506-1509.

Panzieri, S. and G. Ulivi (1995). "Disturbance rejection of Iterative Learning Control Applied to Trajectory for a Flexible Manipulator". In: Proc. ECC 1995. Rome, Italy. pp. 2374-2379. 


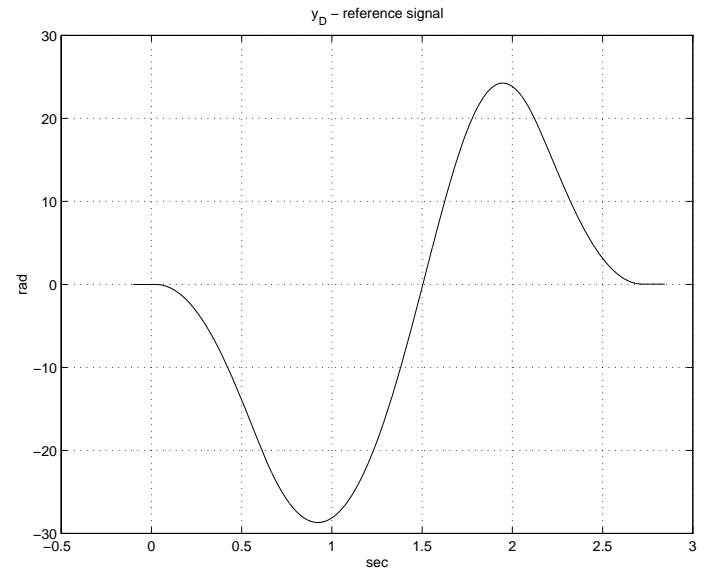

Fig. 2. Reference signal

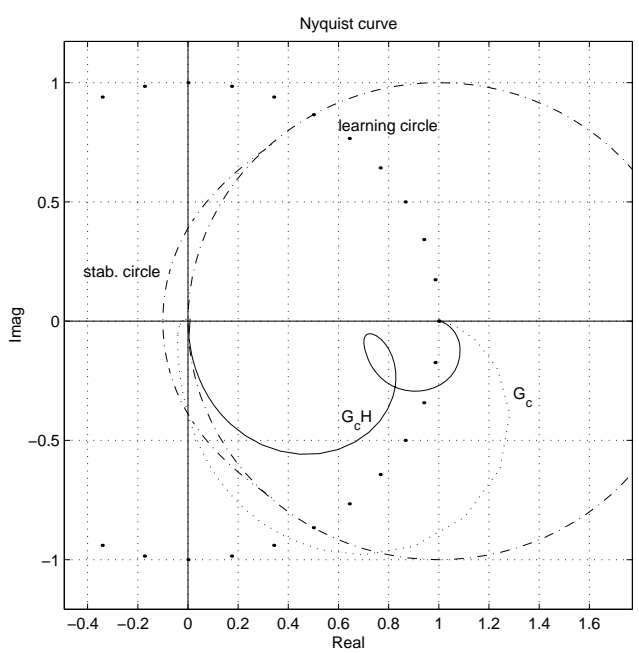

Fig. 3. Nyquist curves for $G_{C} H_{2}$ for the choices $H_{2}=1$ and $H_{2}=\hat{G}_{C}^{-1}\left(1-H_{B}\right)$. Learning circle and stabilizing circle.

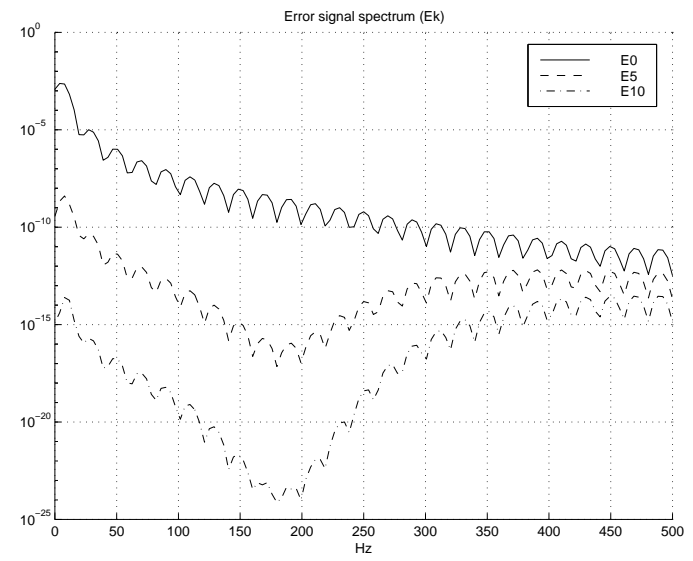

Fig. 4. Error signal spectrum for iterations 0,5 and 10. Linear system. $H_{2}=\hat{G}_{C}^{-1}\left(1-H_{B}\right)$.

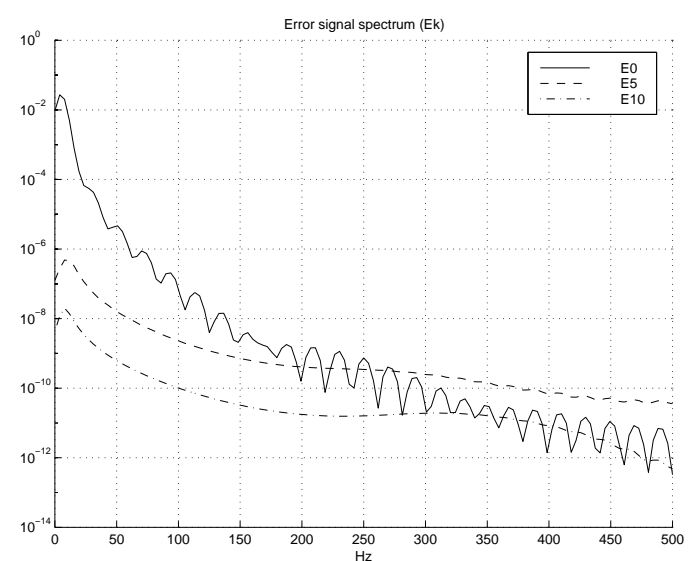

Fig. 5. Error signal spectrum for iterations 0,5 and 10. System with friction. $H_{2}=\hat{G}_{C}^{-1}\left(1-H_{B}\right)$.

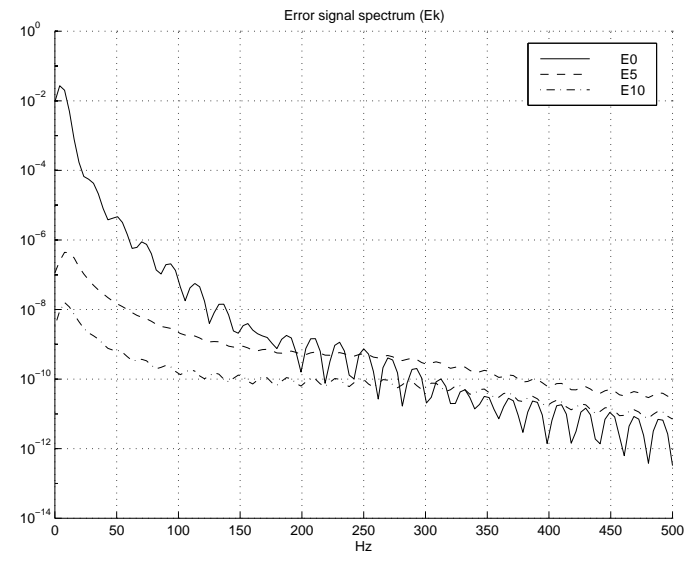

Fig. 6. Error signal spectrum for iterations 0,5 and 10. System with friction. $H=\hat{G}_{C}^{-1}\left(1-H_{B}\right) \cdot V$ high pass filter 